\title{
Morphology and Thermal Characteristics of Polysaccharide Extracted by Ultrasound from Ophiopogon japonicus
}

\author{
Xiaomei Wang1 ${ }^{*}$, Tianzhu Zhang², Wenfei Zhang2, Miao Wang1, Dapeng Hao¹, Qi Ding1 \\ ${ }^{1}$ Faculty of Science, Xi'an Aeronautical University, Xi'an, China \\ ${ }^{2}$ School of Materials Engineering, Xi'an Aeronautical University, Xi'an, China \\ Email: ${ }^{\star} w x m 19830427 @ 163 . c o m$
}

How to cite this paper: Wang, X.M., Zhang, T.Z., Zhang, W.F., Wang, M., Hao, D.P. and Ding, Q. (2018) Morphology and Thermal Characteristics of Polysaccharide Extracted by Ultrasound from Ophiopogon japonicus. American Journal of Plant Sciences, 9, 1699-1707.

https://doi.org/10.4236/ajps.2018.98123

Received: June 19, 2018

Accepted: July 28, 2018

Published: July 31, 2018

Copyright $\odot 2018$ by authors and Scientific Research Publishing Inc. This work is licensed under the Creative Commons Attribution International License (CC BY 4.0).

http://creativecommons.org/licenses/by/4.0/

\begin{abstract}
The crude polysaccharide UPOJ was extracted by ultrasound from Ophiopogon japonicus and purified by DEAE-cellulose 52 column and Sephadex G-150 gel column. Purified polysaccharide UPOJ-DS was obtained. The thermal characteristics of UPOJ-DS were studied by using the thermal analysis system Q1000DSC LNCS FACS Q600SDT. The heating rate was $10^{\circ} \mathrm{C} / \mathrm{min}$ and the test temperature was $20^{\circ} \mathrm{C} \sim 800^{\circ} \mathrm{C}$. The results showed that there were three large mass losses in the process of heating, in which the maximum weight loss rate was $66.68 \%$ at $220^{\circ} \mathrm{C} \sim 330^{\circ} \mathrm{C}$ and the decomposition reaction took place in the polysaccharide itself, which indicated that the composition, water content, intermolecular interaction and aggregation behavior of UPOJ-DS were affected by temperature. The surface morphology of UPOJ-DS was observed by environmental scanning electron microscope (ESEM). The results indicated that the polysaccharide molecules were lamellar or clastic, with smooth surface, strong intermolecular interaction and close binding.
\end{abstract}

\section{Keywords}

Ultrasonic Extraction, Polysaccharide, Thermal Analysis, Environmental Scanning Electron Microscopy (ESEM)

\section{Introduction}

As a traditional Chinese medicine, the plant Ophiopogon japonicus is used for nourishing Yin and nourishing the heart, the treatment of dryness, dry cough, insomnia, constipation, etc [1] [2]. The main chemical constituents are isoflavones, saponins, volatile oils, alcohols, inorganic elements and polysaccharides [3] [4]. The polysaccharide is one of the main active components in Ophiopogon 
japonicus, which has the functions of hypoglycemic activity, anti-fatigue, auxiliary inhibition of tumor, anti-radiation and so on [5] [6] [7]. Ophiopogon japonicus polysaccharide is very important and significant to study its thermal stability and morphology because it has many pharmacological and physiological functions.

Thermal analysis is the technology measuring the relationship of physical properties and temperature dependence under the program control temperature. The commonly used methods include thermogravimetric method (TG), derivative thermogravimetric method (DTG), differential thermal analysis (DTA) and differential scanning calorimetry (DSC). Thermal analysis technique can determine rapidly and accurately the transformation of crystal material type, melting, sublimation, absorption, dehydration and decomposition of change, is an important method to test the physical and chemical properties of inorganic, organic and polymer materials. Thermal analysis technology has been widely used in physics, chemistry, chemical industry, metallurgy, geology, building materials, fuel, textile, food and biology [8] [9] [10].

\subsection{Thermogravimetry (TG) and Derivative Thermogravimetry (DTG)}

Thermogravimetry (TG) is the measurement of the weight change of sample during heating. Temperature is abscissa and the percentage of weight loss is ordinaten in TG curve. Composition, thermal stability, thermal decomposition and products generated could be obtained from TG curve. As well as the temperature range of decomposition and thermal stability could also be obtained from TG curve. The first derivative of the TG curve with respect to temperature is the thermogravimetry (DTG) curve, which reflects the relationship between the rate of change of sample mass and temperature [11].

\subsection{Differential Thermal Analysis (DTA)}

Differential thermal analysis (DTA) is a thermal analysis method to measure the relationship between temperature between samples and reference materials under programmed temperature control [12]. Temperature or time is abscissa and the temperature difference is ordinaten in DTA curve. The physical or chemical processes of samples could be analyzed qualitatively according to the characteristics of differential thermal analysis curves, such as all kinds of endothermic and exothermic peak number, shape, and the corresponding temperature. Reaction heat also can be measured quantitatively according to peak area.

\subsection{Differential Scanning Calorimetry (DSC)}

Differential scanning calorimetry (DSC) is a technique for measuring the relationship between temperature and power difference of material and reference material under programmed temperature control. The DSC curve takes temperature or time as the horizontal coordinate, and the differential of enthalpy with 
respect to time $(\mathrm{dH} / \mathrm{dt})$ as the vertical coordinate. It has the characteristics of sensitive reaction and high resolution [13].

\subsection{Environmental Scanning Electron Microscope (ESEM)}

Environmental Scanning Electron Microscope (ESEM) is a new electronic optical instrument, which uses extremely thin electron beam to scan the sample surface, collects the generated secondary electron with the special detector, forms the electric signal to the picture tube, displays the object on the fluorescent screen. Three-dimensional images of the surface of cells and tissues could be photographed. It has been widely used in physics, chemistry, materials, geology, geography, biology, medicine and other disciplines, and metallurgy, ceramics, electronics, semiconductor and other industries, and promote the development of relevant disciplines, has wide application prospect [14] [15].

\section{Materials and Methods}

\subsection{Materials and Reagents}

Ophiopogon japonicus (produced in Zhejiang province) was purchased from the traditional Chinese medicine market of Wan Shou road, xi' an. Sodium chloride, concentrated sulfuric acid, phenol, $95 \%$ ethanol were all analytical pure reagents. DEAE-52 Whatman, Sephadex G-150 Pharmacia. The experimental water is double steamed water.

\subsection{Instruments and Equipments}

Thermal analysis system (Q1000DSC+LNCS+FACS Q600SDT, TA Co., USA), environmental scanning electron microscope (Quanta200, FEI Co., the Netherlands). Ultrasonic cell crusher (JY92-II, Ningbo Xinzhi Biological Scientific Technology Co., China). Ultraviolet and visible spectrophotometer (TU1810, Beijing's General Instrument Co.). High-speed centrifuge (CF16RX, Hitachi Co., Japan). RE252AA type rotary evaporator, vacuum freezing dryer (FD-1, Beijing Boyikang Laboratory Instrument Co.). $2.5 \times 60 \mathrm{~cm}$ series chromatography column (Shanghai Qite Analytical Instrument Co.) electronic balance (precision: $1 / 10^{5} \mathrm{~g}$, Beijing Sartorius Instrument System Co.).

\subsection{Isolation and Purification of Ophiopogon japonicas Polysaccharide}

The root of Ophiopogon japonicas was crushed after drying at $60^{\circ} \mathrm{C}$, and then $200 \mathrm{~g}$ dry powder was put into $1000 \mathrm{ml}$ beaker, added 4 times the volume of $95 \%$ ethanol to degrease, stirring $20 \mathrm{~min}$, centrifuge, collect precipitate and dry after repeated three times. It was added to distilled water according to the ratio of 1:10 to extract polysaccharide by used of ultrasound. The extraction conditions as follows: ultrasonic power: $80 \mathrm{~W}$, ultrasonic time: $10 \mathrm{~s}$, ultrasonic frequency: 90 times, interval time: $15 \mathrm{~s}$, extract twice, respectively. The twice extract was combined, and added to four times the amount of $95 \%$ ethyl alcohol to precipitate 
polysaccharide after vacuum concentration, then centrifuge, dialyze three days by running water and vacuum freeze-drying. Crude polysaccharide UPOJ was obtained. No absorption was observed at $280 \mathrm{~nm}$ and $260 \mathrm{~nm}$ in the UV absorption spectra of UPOJ, which demonstrated the absence of protein and nucleic acid in the polysaccharides.

The polysaccharide UPOJ was eluted in succession with doubly distilled water by DEAE- 52 cellulose column equilibrated with distilled water $(2.5 \times 60 \mathrm{~cm})$. The eluate was detected by phenol-sulfuric acid method. Only one component obtained, which was named UPOJ-D. The sample of UPOJ-D was purified by Sephadex G-150 gel column $(2.5 \times 60 \mathrm{~cm})$ using doubly distilled water as eluent. Only one component was obtained, named UPOJ-DS. Then UPOJ-DS was concentrated, dialyzed and lyophilized for further study.

\subsection{Thermogravimetry (TG), Differential Thermal Analysis (DTA) and Differential Scanning Calorimetry (DSC)}

With reference to literature [16], the thermal characteristics and aggregation state of Ophiopogon japonicus polysaccharide UPOJ-DS was studied using a thermal analysis system. Test condition: Freeze-dried polysaccharide sample powder was $5-10 \mathrm{mg}$, nitrogen environment (static), test temperature was $20^{\circ} \mathrm{C}$ $-800^{\circ} \mathrm{C}$, heating rate was $10^{\circ} \mathrm{C} / \mathrm{min}$, the scanning speed was $2^{\circ} \mathrm{C} / \mathrm{min}$.

\subsection{Environmental Scanning Electron Microscope (ESEM) Observations}

A proper amount of polysaccharide sample powder was glued to the conductive adhesive of the experimental bench. The floating sample was blown off with rubber suction bulb and placed in a gold conductive layer of $10 \mathrm{~nm}$ thickness in a vacuum plating instrument. The accelerating voltage of the electron gun was $20 \mathrm{KV}$, observed by use of ESEM.

\section{Results and Discussion}

\subsection{DEAE-52 Cellulose Column Chromatography}

Figure 1 showed that only one peak of polysaccharide UPOJ-D was obtained by DEAE-52 cellulose chromatographic column chromatography, indicating that the polysaccharide UPOJ-D was relatively simple.

\subsection{Sephadex G-150 Gel Column Chromatography}

Figure 2 showed that the polysaccharide UPOJ-DS showed a single symmetric peak after the chromatography of Sephadex G-150 gel chromatography, indicating that UPOJ-DS was a polysaccharide with homogeneous components.

\subsection{TG-DTG Analysis of UPOJ-DS}

As shown in Figure 3, dry UPOJ-DS had three big mass loss from $20^{\circ} \mathrm{C}$ to $800^{\circ} \mathrm{C}$ in the process of temperature rising. The weight loss ratio was $7.774 \%$ in the 


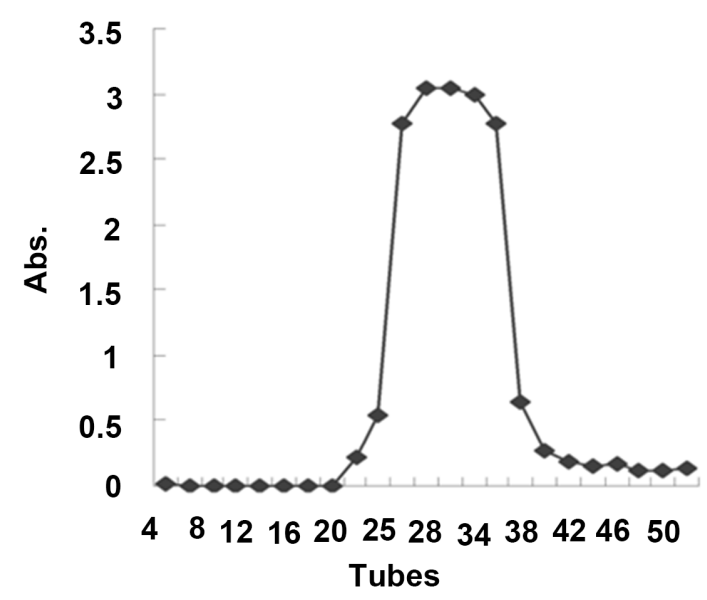

Figure1. DEAE-52 cellulose column chromatography of UPOJ.

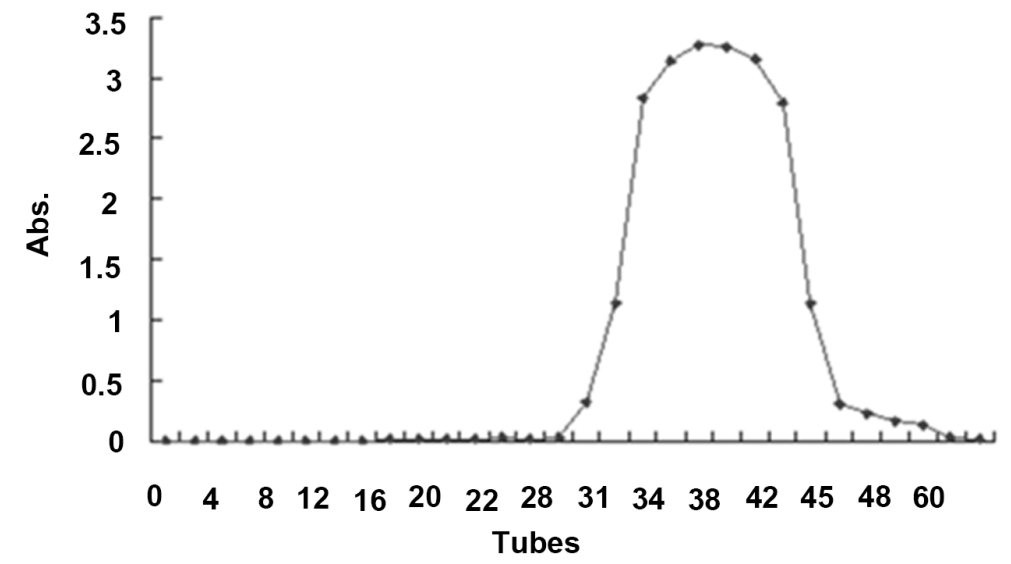

Figure 2. Sephadex G-150 gel column chromatography of UPOJ-D.

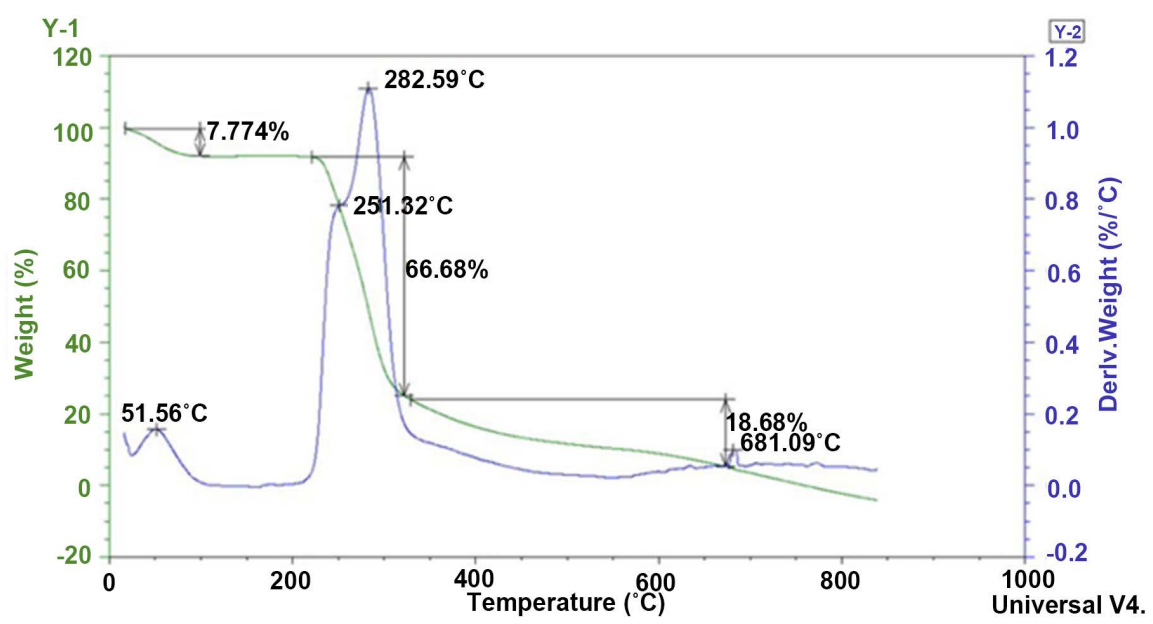

Figure 3. The TG-DTG curves of UPOJ-DS.

process of $20^{\circ} \mathrm{C} \sim 100^{\circ} \mathrm{C}$, which was the process of loss of the adsorption of water. In $220^{\circ} \mathrm{C} \sim 330^{\circ} \mathrm{C}$, the weight loss ratio was $66.68 \%$, suggesting that poly- 
saccharide decomposition reaction happened. In $330^{\circ} \mathrm{C} \sim 700^{\circ} \mathrm{C}$, the weight loss ratio was $18.68 \%$. Thereafter until heated to $800^{\circ} \mathrm{C}$, the weight of UPOJ-DS had been continuously decreasing, but the weight loss ratio significantly smaller than before. These results indicated that these change of UPOJ-DS was related to its composition, water content, molecular interaction and aggregation behavior. DTG curves reflected the relationship between the mass-change rate and temperature. The mass-change rate also has two peaks in Figure 3. It had a higher peak and a greater weight loss rate at $220^{\circ} \mathrm{C} \sim 330^{\circ} \mathrm{C}$. The weight loss rate was largest at $283^{\circ} \mathrm{C}$ especially.

\subsection{DTA and DSC Analysis}

Figure 4 and Figure 5 were DTA and DSC analysis charts of UPOJ-DS respectively. From Figure 4, there were two endothermic peaks. The weight of sample was decreased from $99.36 \%$ to $91.96 \%$ in the first peak at $21^{\circ} \mathrm{C} \sim 110^{\circ} \mathrm{C}$. This was the process of water evaporates and absorbs heat. The second peak was the heat absorbed by polysaccharide decomposition at $230^{\circ} \mathrm{C} \sim 330^{\circ} \mathrm{C}$, which was consistent with the TG analysis results. From Figure 5, the heat of sample absorption was in two stages. The first stage began to heat absorption at $20^{\circ} \mathrm{C}$ and stopped heat absorption at $65^{\circ} \mathrm{C}$, and the weight of sample was decreased in the process. It was caused by losing adsorption water known by the preceding analysis. The second stage, there was a peak in $233^{\circ} \mathrm{C} \sim 330^{\circ} \mathrm{C}$. In this process, the sample absorbed heat and underwent a thermal change. The key indicators of the thermal changes were as follows: extrapolation starting temperature was $233.11^{\circ} \mathrm{C}$, extrapolation end temperature was $328.37^{\circ} \mathrm{C}$, extreme value was $283.63^{\circ} \mathrm{C}$, the peak height flow was $8.526 \mathrm{w} / \mathrm{g}$, enthalpy value was $211.3 \mathrm{~J} / \mathrm{g}$. These results were consistent with the literature [17]. It indicated that UPOJ-DS has good thermal stability.

\subsection{Environmental Scanning Electron Microscope (ESEM) Observations of UPOJ-DS}

Figure 6(a), Figure 6(b), and Figure 6(c) were the electron microscope scanning photos of polysaccharide UPOJ-DS at different magnification multiples. The results showed that the sample was lamellar or clastic and the surface was smooth. The curly shape, the smooth sheet surface and holes could be clearly observed at 500 of magnification multiples. At high magnification multiples, the surface was relatively compact, indicating that the polysaccharide molecules interact strongly with each other and bind closely.

\section{Conclusion}

The above conclusion indicated that the molecular structure of Ophiopogon japonicas polysaccharide UPOJ-DS will change with the change of temperature. The experiments of TG, DTA and DSC were used to study the thermal characteristics of UPOJ-DS. The analysis results showed that the UPOJ-DS was relatively 


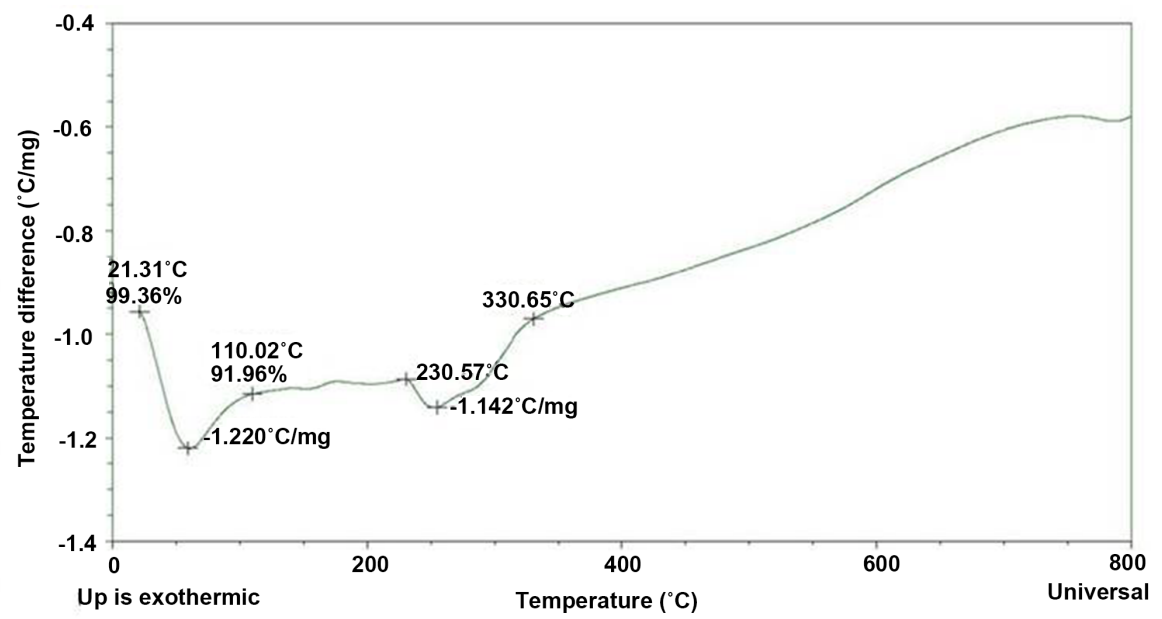

Figure 4. The DTA curves of UPOJ-DS.

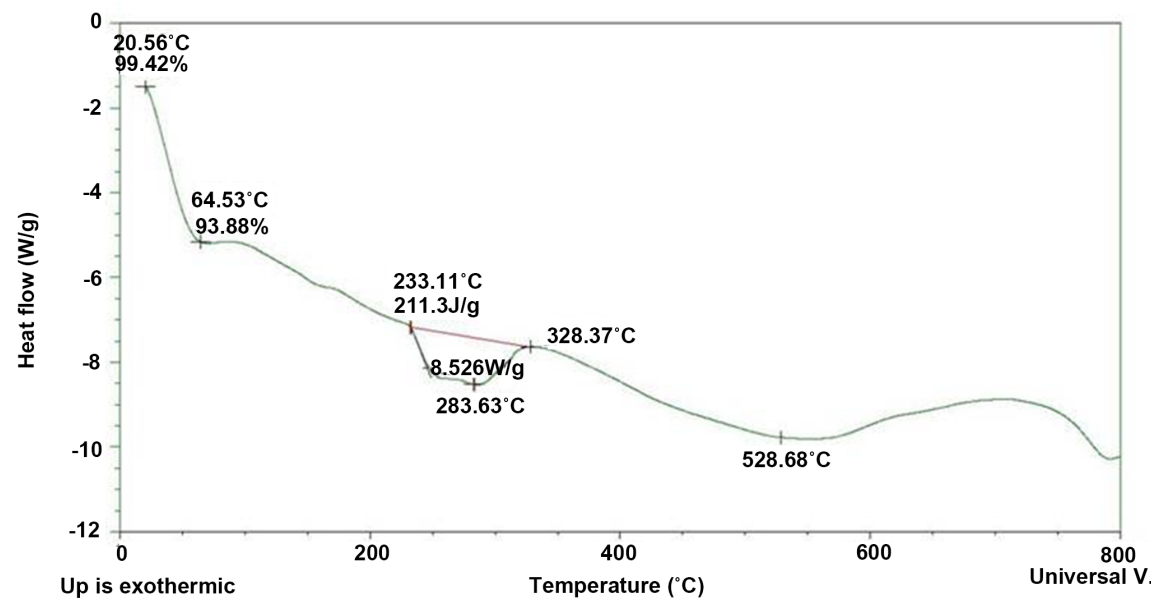

Figure 5. The DSC curves of UPOJ-DS.

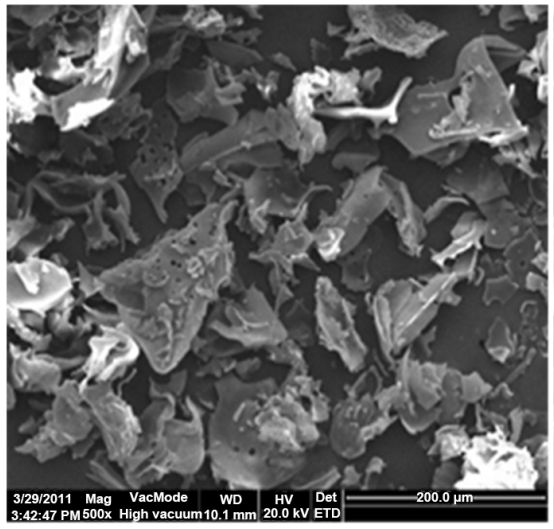

(a)

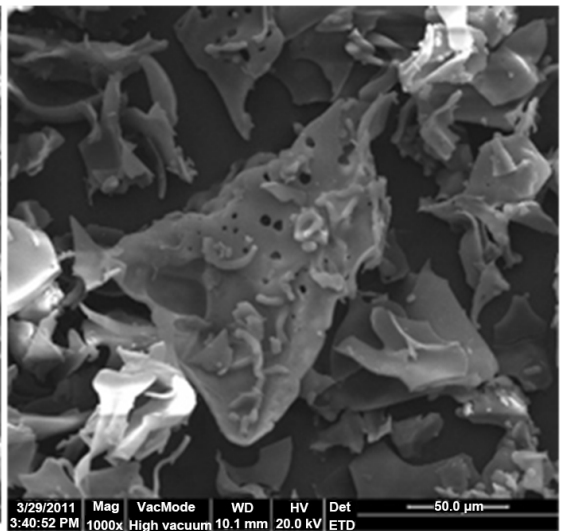

(b)

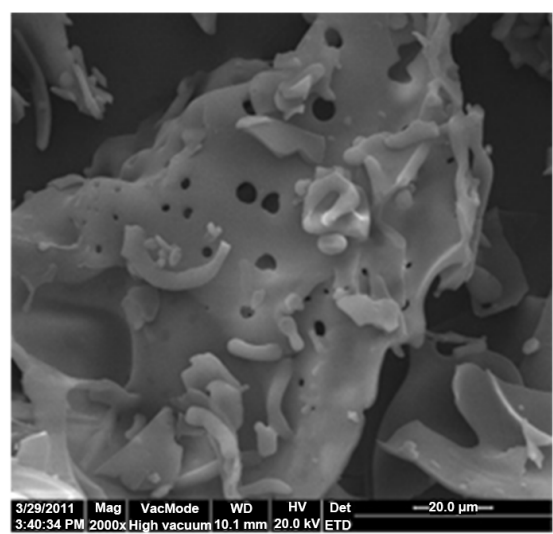

(c)

Figure 6. The electron microscope scanning photos of UPOJ-DS. (a: 500x; b:1000x; c: 2000x).

stable under $200^{\circ} \mathrm{C}$, and rapid decomposition occurred at about $280^{\circ} \mathrm{C}$. There were three times mass loss and two endothermic peaks in the heating process, suggesting the composition, water content and the intermolecular of UPOJ-DS 
were affected by temperature. It resulted in the change of the state of aggregation behavior of UPOJ-DS. From several above experiments, we could know the DTG curve is the first derivative of the TG curve with respect to temperature, enhanced the accuracy and could accurately determine the changes of thermogravimetric process. The DSC analysis was used to calculate quantitatively accurately, and was more sensitive, high resolution, and good reproducibility. But the highest determination temperature of DSC was only about $750^{\circ} \mathrm{C}$, differential thermal analysis method could be used when determination temperature was higher than the temperature $750^{\circ} \mathrm{C}$. The scanning electron microscopy showed that the polysaccharide molecules were lamellar or clastic, with smooth surface, strong intermolecular interaction and close binding. In conclusion, the results provided theoretical and experimental foundation for the thermal properties and morphology of the polysaccharide extracted by ultrasound from Ophiopogon japonicas.

\section{Acknowledgements}

This research was financially supported by the Natural Science Foundation of Shaanxi Province (Grant Numbers, 2018JQ1091); the Fundamental Research Funds for Xi'an Aeronautical University (Grant Numbers, 2016KY1215); undergraduate innovation and entrepreneurship training program of Xi'an Aeronautical University (Grant Numbers, DCX2018042).

\section{References}

[1] Kou, J., Sun, Y., Lin, Y., Cheng, Z., Zheng, W., Yu, B. and Xu, Q. (2005) Anti-Inflammatory Activities of Aqueous Extract from Radix Ophiopogon japonicus and Its Two Constituents. Biological and Pharmaceutical Bulletin, 28, 1234-1238. https://doi.org/10.1248/bpb.28.1234

[2] Hung, T.M., Van Thu, C., Dat, N.T., Ryoo, S.W., Lee, J.H., Kim, J.C., Na, M., Jung H.J., Bae, K.H. and Min, B.S. (2010) Homoisoflavonoid Derivatives from the Roots of Ophiopogon japonicus and Their in Vitro Anti-Inflammation Activity. Bioorganic \& Medicinal Chemistry Letters, 20, 2412-2416.

https://doi.org/10.1016/j.bmcl.2010.03.043

[3] Kou, J., Tian, Y., Tang, Y., Yan, J. and Yu, B. (2006) Antithrombotic Activities of Aqueous Extract from Radix Ophiopogon japonicus and Its Two Constituents. Biological and Pharmaceutical Bulletin, 29, 1267-1270.

https://doi.org/10.1248/bpb.29.1267

[4] Li, N., Zhang, J.Y., Zeng, K.W., Zhang, L., Che, Y.Y. and Tu, P.F. (2012) Anti-Inflammatory Homoisoflavonoids from the Tuberous Roots of Ophiopogon japonicus. Fitoterapia, 83, 1042-1045. https://doi.org/10.1016/j.fitote.2012.05.011

[5] Fang, J., Wang, X., Lu, M., He, X. and Yang, X. (2018). Recent Advances in polysaccharides from Ophiopogon japonicus and Liriopespicata var. Prolifera. International Journal of Biological Macromolecules, 114, 1257-1266. https://doi.org/10.1016/j.ijbiomac.2018.04.022

[6] Fan, Y., Ma, X., Ma, L., Zhang, J., Zhang, W. and Song, X. (2016) Antioxidative and Immunological Activities of Ophiopogon Polysaccharide Liposome from the Root 
of Ophiopogon japonicus. Carbohydrate Polymers, 135, 110-120. https://doi.org/10.1016/j.carbpol.2015.08.089

[7] Zhang, J., Fan, S., Mao, Y., Ji, Y., Jin, L., Lu, J. and Chen, X. (2016) Cardiovascular Protective Effect of Polysaccharide from Ophiopogon japonicus in Diabetic Rats. International Journal of Biological Macromolecules, 82, 505-513. https://doi.org/10.1016/j.ijbiomac.2015.09.069

[8] Grabowska, B., Kaczmarska, K., Bobrowski, A., Żymankowska-Kumon, S. and Kurleto-Kozioł, Ż. (2017) TG-DTG-DSC, FTIR, DRIFT and Py-GC-MS Studies of Thermal Decomposition for poly (sodium acrylate)/dextrin (PAANa/D)-New Binder BioCo3. Journal of Casting \& Materials Engineering, 1, 27. https://doi.org/10.7494/jcme.2017.1.1.27

[9] Skwarek, E., Goncharuk, O., Sternik, D., Janusz, W., Gdula, K. and Gun'ko, V. M. (2017) Synthesis, Structural, and Adsorption Properties and Thermal Stability of Nanohydroxyapatite/Polysaccharide Composites. Nanoscale Research Letters, 12, 155. https://doi.org/10.1186/s11671-017-1911-5

[10] Horvat, G., Fajfar, T., Uzunalić, A.P., Knez, Ž. and Novak, Z. (2017) Thermal Properties of Polysaccharide Aerogels. Journal of Thermal Analysis and Calorimetry, 127, 363-370. https://doi.org/10.1007/s10973-016-5814-y

[11] Dollimore, D., Evans, T.A., Lee, Y.F. and Wilburn, F.W. (1992) Correlation between the Shape of a TG/DTG Curve and the Form of the Kinetic Mechanism Which Is Applying. Thermochimicaacta, 198, 249-257. https://doi.org/10.1016/0040-6031(92)85081-6

[12] Anbukarasi, K. and Kalaiselvam, S. (2015) Study of Effect of Fibre Volume and Dimension on Mechanical, Thermal, and Water Absorption Behaviour of Luffa Reinforced Epoxy Composites. Materials \& Design (1980-2015), 66, 321-330. https://doi.org/10.1016/j.matdes.2014.10.078

[13] Pani, N.R., Nath, L.K., Acharya, S. and Bhuniya, B. (2011) Application of DSC, IST, and FTIR Study in the Compatibility Testing of Nateglinide with Different Pharmaceutical Excipients. Journal of Thermal analysis and Calorimetry, 108, 219-226. https://doi.org/10.1007/s10973-011-1299-x

[14] Stabentheiner, E., Zankel, A. and Pölt, P. (2010) Environmental Scanning Electron microscopy (ESEM) -A Versatile Tool in Studying Plants. Protoplasma, 246, 89-99. https://doi.org/10.1007/s00709-010-0155-3

[15] Wu, W., Zhu, Y., Zhang, L., Yang, R. and Zhou, Y. (2012) Extraction, Preliminary Structural Characterization, and Antioxidant Activities of Polysaccharides from Salvia miltiorrhiza Bunge. Carbohydrate Polymers, 87, 1348-1353. https://doi.org/10.1016/j.carbpol.2011.09.024

[16] Bothara, S.B. and Singh, S. (2012) Thermal Studies on Natural Polysaccharide. Asian Pacific Journal of Tropical Biomedicine, 2, S1031-S1035. https://doi.org/10.1016/S2221-1691(12)60356-6

[17] Chen, L. Liu, J., Zhang, Y., Dai, B., An, Y. and Yu, L. (2015) Structural, Thermal, and Anti-Inflammatory Properties of a Novel Pectic Polysaccharide from Alfalfa (Medicago sativa L.) Stem. Journal of Agricultural and Food Chemistry, 63, 3219-3228. https://doi.org/10.1021/acs.jafc.5b00494 